Klägerin gelieferte Anwendersoftware ist mangelhaft, weil das dazu gelieferte Bedienungshandbuch unvollständig ist. Das ergibt sich aus dem überzeugenden Gutachten des Sachverständigen. Dieser hat einleuchtend ausgeführt, daß ein Programm dieser Ansprüche ein Bedienungshandbuch erfordert, welches nicht durch mündliche Einweisungen ersetzt werden kann. Ohne ein solches Handbuch ist daher das gelieferte Anwenderprogramm für die Beklagte nicht ordnungsgemäß nutzbar.

Dieser Mangel ist auch nicht nach $\S 377$ Abs. 2 oder Abs. 3 HGB genehmigt. Bei der Lieferung der Anwendersoftware und des sogenannten vorläufigen Bedienungshandbuches konnte die Beklagte auch bei einer in ordnungsgemäßem Geschäftsgang tunlichen Untersuchung nicht feststellen, ob das Bedienungshandbuch für den Betrieb der Anlage ausreichte. Es entspricht der Lebenserfahrung, daß die Qualität eines Handbuches für die Arbeit mit Anwendersoftware erst im Laufe des Betriebes der Computeranlage festgestellt werden kann. Daher ist davon auszugehen, daß die Beklagte erst bei Abfassung ihres Schreibens vom 12. 8 . 1985 die Unbrauchbarkeit des gelieferten Programmpaketes in dem bisher gelieferten Umfang erkannt hat. Zwar hat die Beklagte mit diesem Schreiben die Unvollständigkeit des Bedienungshandbuches nicht beanstandet. Da das Bedienungshandbuch aber nach dem Sachverständigengutachten für die ordnungsgemäße Nutzbarkeit der Anwendersoftware erforderlich war, die Beklagte andererseits nicht nur bestimme Beispiele für Fehler aufgeführt, sondern allgemein die Nutzbarkeit des Programms beanstandet hat, bezog sich diese Rüge auch auf Fehler des Bedienungshandbuches. Außerdem hat die Klägerin durch ihr Schreiben vom 19. 8. $1985 \mathrm{zu}$ erkennen gegeben, daß sie Schwierigkeiten bei der Arbeit der Beklagten mit dem Programm unter anderem auf einen mangelnden Informationsfluß zurückführte. Auch für die Klägerin war demnach erkennbar, daß Bedienungsprobleme für die Beklagte Anlaß zu ihrem Schreiben vom 12. 8. 1985 waren und damit auch Fehler des Bedienungshandbuches für die Beanstandung mitursächlich sein konnten. Unter diesen Umständen bezieht sich das Schreiben vom 12. 8. 1985 auch auf etwaige Mängel des Bedienungshandbuches. Durch eine Genehmigung nach $\S 377$ Abs. 3 HGB war daher das Wandelungsrecht der Beklagten nicht ausgeschlossen.

Die Mängeleinrede der Beklagten ist auch nicht nach $\S 477$ BGB verjährt. Wegen der 12 monatigen Garantiefrist für die Software begann der Lauf der 6 monatigen Verjährungsfrist erst 12 Monate nach der Lieferung der Software, also mit Ablauf des 17. 5. 1986. Daher ist sowohl die Beanstandung vom 12. 8. 1985 als auch speziell für das Bedienungshandbuch vom 10.11. 1986 noch innerhalb der Verjährungszeit erfolgt. Damit ist die Wandelungseinrede für die Beklagte gem. $\S 478$ BGB erhalten geblieben....

(Einsendung:

Rechtsanwalt Dr. Christoph Zabrnt, Neckargemünd)

\title{
Systemtechnische Dokumentation
}

\section{AG Pforzheim, Urteil vom 7. Juli 1987 (3 C 540/86)}

\section{Nichtamtlicher Leitsatz}

Wer die Lieferung von Quellcode schuldet, hat auch eine systemtechnische Dokumentation zu liefern.

\section{Paragraphen}

BGB: $\S 631$

\section{Stichworte}

Programmerstellungsvertrag - geschuldete systemtechnische Dokumentation

\section{Tatbestand}

„Der Kläger hatte für den Beklagten Software erstellt. Nach Beendigung der Geschäftsbeziehungen vereinbarten die Parteien, daß der Kläger dem Beklagten (den) Source-Code“ (=Quellcode) „gegen Zahlung von $3500,-$ DM herausgab. Er übergab auch den Source-
Code dem Beklagten. Mit der Klage verlangt er Zahlung der vereinbarten 3500,- DM. “ Der Beklagte wendet ein, daß der Quellcode nicht dokumentiert sei.

\section{Entscheidungsgründe}

„Die Klage ist nicht begründet. Der vom Kläger dem Beklagten übergebene Source-Code entspricht nicht den Anforderungen, die an einen solchen gestellt werden müssen und ist auch für Fachleute nicht ohne weiteres verwendbar. Er ist deshalb mangelhaft. Bis zur Behebung dieser Mängel ist der Beklagte berechtigt, den vereinbaren Preis zurückzubehalten.

Dies ergibt sich eindeutig aus dem überzeugenden Gutachten des Sachverständigen. ... Ein Programm bedarf, um auch für einen Fachmann verständlich und verwertbar zu sein, der Dokumentation, wie es auch die DIN-Norm 66230 sowohl im allgemeinen Teil unter Ziffer 1 und 3 als auch in der Ziffer 2.2.1 bestimmt. 
Der Hinweis des Klägers, diese DIN-Norm sei nicht vereinbart, geht fehl. Die DIN-Norm braucht nicht besonders vereinbart zu werden, sondern eine DINNorm bezeichnet die nach dem Stand ihres Erlasses (hier 1981) allgemein übliche und erforderliche Beschaffenheit. Hier fällt auf, daß der Kläger über $140 \times$ das Wort ,Rem' ohne Zusatz verwendet, wobei das Wort Rem allein sinnlos ist und nur zur Einleitung eines darauffolgenden Kommentars dient....

\section{Anmerkung}

Zum Begriff Quellcode (,source code") siehe Zahrnt, DV-Verträge: Rechtsfragen und Rechtsprechung, S. $28 \mathrm{ff}$.
Die Sachverständigen gehen in ihren Gutachten durchweg davon aus, daß die systemtechnische Dokumentation zum Lieferumfang gehört. Das ist bisher nicht so deutlich wie hier in einem Urteil zum Ausdruck gekommen, weil das nie im Zentrum der Entscheidung stand.

Das Urteil übersieht, daß Quellcode bei Programmerstellung ohnehin zur geschuldeten Leistung gehört (vgl. Zahrnt, a. a. O., S. 254).

(Einsendung und Anmerkung: Rechtsanwalt Dr. Cbristoph Zabrnt, Neckargemünd)

\section{Software}

\section{„Hexengriffel“" für Juristen? - Das Textverarbeitungsprogramm „Witchpen“}

\section{Michael Zurek}

Das von Hannes Keller gegründete Schweizer Software-Haus "Witch-Systems ${ }^{\star}$ hat mit WITCHPEN ein Textverarbeitungsprogramm entwickelt, das wegen seines günstigen Preises für Studenten interessant ist und daneben mit seinem „Blitzwörterbuch" auch in juristischen Arbeitsumgebungen einige nützliche Anwendungsmöglichkeiten bietet. Hinzu kommt für WORDSTAR-erfahrene Benutzer ein weiterer Auswahlgesichtspunkt: Das Menü am oberen Bildrand ist nicht nur sehr ähnlich, sondern auch manche Control-Befehle sind identisch. Denjenigen, die das weit verbreitete Programm WORDSTAR bereits kennen, bereitet es keine Probleme, mit WITCHPEN zu arbeiten.

\section{Hardware-Voraussetzungen}

WITCHPEN wird in der Grundversion auf drei 5 1/4 Zoll Disketten im MS-DOS-Format geliefert. Es benötigt als Minimum einen IBM PC/XT/AT (oder vollständig dazu kompatiblen Rechner) mit zwei Diskettenlaufwerken (besser Festplatte), das Betriebssystem PC-/MS-DOS 2.0 oder eine spätere Version sowie $256 \mathrm{KByte}$ (vorzugsweise $512 \mathrm{KByte}$ ) Arbeitsspeicher. Farbgrafikkarten werden unterstützt. Das Programm ist nicht mit einem Kopierschutz versehen, was die Installation erleichtert.

Die Funktionen von WITCHPEN beschränken sich in der getesteten Version auf das im folgenden beschriebene Grundmodul zum Preis von 112,- DM (inklusive Mehrwertsteuer und Versand).

Getestet wurde das Programm auf einem Commodore PC10 mit Festplatte. Der Versuch, das Programm auch auf dem Laptop NEC Multispeed zu testen, schlug fehl. WITCHPEN stürzte regelmäßig ab, so daß der Computer nur noch mit einem Kaltstart wieder zum Leben erweckt werden konnte. Auch ein zweiter zum Vergleich herangezogener NEC Multispeed zeigte trotz großer Bemühungen das gleiche Bild. Dieses Verhalten ist wahrscheinlich auf den geänderten Hardwareaufbau des Nec Multispeed zurückzuführen. Anstelle des Intel 8086 wurde ein modifizierter Mikroprozessor mit der Bezeichnung NEC V30 verwendet.

Gegen Einsendung von 10,- DM erhält man bei der Firma Witch-Systems eine Demo-Diskette, mit der man überprüfen kann, ob das Programm auf dem betreffenden Computer lauffähig ist. Gleichzeitig wird dem Anwender auch ein Überblick über die Funktionen von WITCHPEN vermittelt.

\section{Installation}

$\mathrm{Zu}$ Beginn ist es erforderlich, das Programm auf dem Computer zu installieren. Dazu werden alle Dateien des Programmes in ein Unterverzeichnis auf der Festplatte mit dem PC-/MS-DOS-Kommando 〈A: COPY ${ }^{*}{ }^{*} \mathrm{C}$ :) kopiert. Ist eine solche nicht vorhanden, so kann auch mit einem Zwei-Diskettenlaufwerk-System gearbeitet werden. In dem Fall empfiehlt es sich, unbedingt Arbeitskopien der Original-Disketten mit dem PC-/MS-DOS-Kommando (DISKCOPY A: B:) anzufertigen. Eine automatische Installierung ist nicht vorgesehen, so daß der PC-/MS-DOS Unerfahrene hier bereits auf erste Schwierigkeiten stoßen kann. 